\title{
ON THE UNIQUENESS OF SOLUTIONS OF CERTAIN DIOPHANTINE EQUATIONS
}

\author{
JOSEPH B. MUSKAT AND YUN-CHENG ZEE
}

\begin{abstract}
An arithmetic proof by L. E. Dickson of the uniqueness of the integral solutions of a certain quaternary quadratic form is generalized to include several similar forms which have appeared recently in cyclotomy.

In his exposition of cyclotomy of order 5, Dickson studied the quater-
\end{abstract} nary quadratic form

$$
16 p=x^{2}+125 w^{2}+50 u^{2}+50 v^{2}
$$

with the side condition

$$
x w=v^{2}-u^{2}-4 u v,
$$

where $p$ is any prime $\equiv 1(\bmod 5)$. He gave an arithmetic proof, and an algebraic proof, that (1) and (2) have essentially a unique integral solution.

There are eight related solutions; if $(x, w, u, v)$ is a solution, so are $(x, w$, $-u,-v),(x,-w, v,-u)$, and $(x,-w,-v, u)$. The other four are obtained from these by reversing all the signs [1, Theorem 8 ].

In recent studies of cyclotomy there have appeared several other examples of forms

$$
k p=c_{1} x^{2}+c_{2} w^{2}+c_{3} u^{2}+c_{4} v^{2}, \quad c_{1}, c_{2}, c_{3}, c_{4}>0,
$$

$p$ any odd prime $\equiv a(\bmod e)$, with the side condition

$$
d_{1} x w=d_{2} v^{2}+d_{3} u^{2}+d_{4} u v
$$

where all coefficients are relatively prime to $p$. The symbols $x, w, u$ and $v$ denote rational linear combinations of coefficients of Jacobi sums (if $a=1$ ) or Eisenstein sums; $k$ is taken to be greater than 1 where necessary to insure that $x, w, u$ and $v$ are integers. Thus for each pair $(e, a)$ to be considered here, there are integers $c_{1}, c_{2}, c_{3}, c_{4}, d_{1}, d_{2}, d_{3}, d_{4}$ (which depend only on $e$ and $a)$ such that for every $p \equiv a(\bmod e)$, (3) and (4) are solvable in integers.

Received by the editors November 15, 1973 and, in revised form, February 15, 1974.

AMS (MOS) subject classifications (1970). Primary 10B05, $10 \mathrm{C} 05$.

Key words and phrases. Cyclotomy, quaternary quadratic form. 
In this paper, we generalize Dickson's arithmetic proof to show that a number of these forms have essentially unique integral solutions.

First we express $u$ and $v$ linearly in terms of $x$ and $w$. All congruences will be modulo $p$.

Assume that the following five restrictions are satisfied by the coefficients in (3) and (4).

$$
\left[-1 \pm 2 s d_{2} /\left(c_{3} d_{1}\right)\right] c_{1} / c_{3} \text { are quadratic residues of } p,
$$

where $s$ satisfies $s^{2} \equiv c_{1} c_{2}$.

Transpose the last term in (4), apply (6), and square:

$$
\begin{aligned}
& d_{2}^{2}\left(v^{2}-u^{2}\right)^{2}=\left(d_{1} x w-d_{4} u v\right)^{2}, \\
& d_{2}^{2}\left(v^{2}+u^{2}\right)^{2}=\left(d_{1} x w-d_{4} u v\right)^{2}+4 d_{2}^{2} u^{2} v^{2} .
\end{aligned}
$$

Transpose (3), regarded as a congruence $(\bmod p)$, and apply (5):

$$
c_{3}\left(v^{2}+u^{2}\right) \equiv-c_{1} x^{2}-c_{2} w^{2} .
$$

Substitute (11) into (10):

$$
\begin{aligned}
d_{2}^{2}\left(-c_{1} x^{2}-c_{2} w^{2}\right)^{2} \equiv & c_{3}^{2}\left(d_{1} x w-d_{4} u v\right)^{2}+4 c_{3}^{2} d_{2}^{2} u^{2} v^{2}, \\
d_{2}^{2}\left(c_{1} x^{2}-c_{2} w^{2}\right)^{2} \equiv & \left(c_{3}^{2} d_{1}^{2}-4 c_{1} c_{2} d_{2}^{2}\right) x^{2} w^{2} \\
& -2 c_{3}^{2} d_{1} d_{4} x w u v+c_{3}^{2}\left(d_{4}^{2}+4 d_{2}^{2}\right) u^{2} v^{2} \\
& \equiv x^{2} w^{2} c_{1} c_{2} d_{4}^{2}-2 x w u v c_{3}^{2} d_{4} d_{1}+u^{2} v^{2} c_{3}^{4} d_{1}^{2} /\left(c_{1} c_{2}\right),
\end{aligned}
$$

upon two applications of (7),

$$
\equiv\left(x w c_{1} c_{2} d_{4}-u v c_{3}^{2} d_{1}\right)^{2} /\left(c_{1} c_{2}\right) .
$$

Take the square root and separate $u v$, choosing the sign of $s$ appropriately:

$$
c_{3}^{2} d_{1} u v \equiv c_{1} c_{2} d_{4} x w+s d_{2}\left(c_{1} x^{2}-c_{2} w^{2}\right) .
$$


Add $2 /\left(c_{3} d_{1}\right)$ times (12) to (11):

$$
\begin{aligned}
c_{3}\left(v^{2}+2 u v+u^{2}\right) & \equiv c_{1} x^{2}\left(-1+2 s d_{2} /\left(c_{3} d_{1}\right)\right) \\
& +2 x w c_{1} c_{2} d_{4} /\left(c_{3} d_{1}\right)+c_{2} w^{2}\left(-1-2 s d_{2} /\left(c_{3} d_{1}\right)\right) .
\end{aligned}
$$

In view of (9), define

$$
\begin{gathered}
m^{2} \equiv\left[-1+2 s d_{2} /\left(c_{3} d_{1}\right)\right] c_{1} / c_{3}, \\
t^{2} \equiv\left[-1-2 s d_{2} /\left(c_{3} d_{1}\right)\right] c_{1} / c_{3} . \\
m^{2} t^{2} \equiv\left[1-4 c_{1} c_{2} d_{2}^{2} /\left(c_{3}^{2} d_{1}^{2}\right)\right] c_{1}^{2} / c_{3}^{2} \equiv\left[s c_{1} d_{4} /\left(c_{3}^{2} d_{1}\right)\right]^{2},
\end{gathered}
$$

by (7). Having picked $m$, choose the sign of $t$ so that

$$
m t \equiv s c_{1} d_{4} /\left(c_{3}^{2} d_{1}\right)
$$

The congruences

$$
m^{2}+t^{2} \equiv-2 c_{1} / c_{3}, \quad m^{2}-t^{2} \equiv 4 s c_{1} d_{2} /\left(c_{3}^{2} d_{1}\right)
$$

are noted here for later reference. Now (13) can be written as

$$
\begin{gathered}
v^{2}+2 u v+u^{2} \equiv x^{2} m^{2}+2 x w m t s / c_{1}+w^{2} t^{2} c_{2} / c_{1} \\
v+u \equiv q_{1}\left(x m+w t s / c_{1}\right), \quad q_{1}^{2}=1 .
\end{gathered}
$$

Similarly, subtracting $2 /\left(c_{3} d_{1}\right)$ times (12) from (11) yields, after simplification,

$$
\begin{gathered}
v^{2}-2 u v+u^{2} \equiv x^{2} t^{2}-2 x w m t s / c_{1}+w^{2} m^{2} c_{2} / c_{1} \\
v-u \equiv q_{2}\left(x t-w m s / c_{1}\right), \quad q_{2}^{2}=1 .
\end{gathered}
$$

Thus

$$
(v+u)(v-u) \equiv q_{1} q_{2}\left[\left(c_{1} x^{2}-c_{2} w^{2}\right) s_{4}-4 x w c_{1} c_{2} d_{2}\right] /\left(c_{3}^{2} d_{1}\right),
$$

by (15) and (16). Apply (12) and regroup:

$$
\begin{aligned}
v^{2}-u^{2} & \equiv q_{1} q_{2}\left[u v d_{4} / d_{2}-x w c_{1} c_{2}\left(d_{4}^{2}+4 d_{2}^{2}\right) /\left(c_{3}^{2} d_{1} d_{2}\right)\right] \\
& \equiv q_{1} q_{2}\left[d_{4} u v-d_{1} x w\right] / d_{2},
\end{aligned}
$$

by (7). Now apply (4) and (6):

$$
v^{2}-u^{2} \equiv-q_{1} q_{2}\left(v^{2}-u^{2}\right)
$$

Hence if $v^{2} \equiv u^{2}, q_{2}=-q_{1}$. If $v^{2} \equiv u^{2}$, one of $q_{1}$ and $q_{2}$ can be chosen arbitrarily; choose that one so that $q_{2}=-q_{1}$. (The latter situation actually occurs.) In either case, 


$$
\begin{aligned}
& 2 v \equiv q_{1}\left[x(m-t)+w(t+m) s / c_{1}\right], \\
& 2 u \equiv q_{1}\left[x(m+t)+w(t-m) s / c_{1}\right] .
\end{aligned}
$$

But $q_{1}$ can be discarded, for the effect of changing the sign of $q_{1}$ can be achieved by changing the signs of both $m$ and $t$. Thus

$$
\begin{aligned}
& 2 v \equiv x(m-t)+w(t+m) s / c_{1}, \\
& 2 u \equiv x(m+t)+w(t-m) s_{1} / c_{1} .
\end{aligned}
$$

Thus there are two signs to be chosen, those of $s$ and $m$.

Let $(x, w, u, v)$ be a solution of (3) and (4). $(-x,-w,-u,-v)$ is another. Changing the signs of $m$ and $t$ gives two more, $(x, w,-u,-v)$ and $(-x,-w, u, v)$. Changing the sign of $s$ interchanges $m^{2}$ with $t^{2}$, and the sign of the product $m t$ is changed (see (14) and.(15)). Thus replacing $s, w$, $m, t$ by $-s,-w, t,-m$ in (17) gives the solution $(x,-w, v,-u)$. The other three solutions are obtained by changing the signs of $m$ and $t$ or $x$ and $w$.

Fix the signs of $s$ and $m$ in (17) and let

$$
(x, w, u, v),\left(x^{\prime}, w^{\prime}, u^{\prime}, v^{\prime}\right)
$$

be two solutions. We have

$$
\begin{aligned}
4 v v^{\prime} & \equiv x x^{\prime}(m-t)^{2}+w w^{\prime}(t+m)^{2} c_{2} / c_{1}+\left(x w^{\prime}+x^{\prime} w\right)\left(m^{2}-t^{2}\right) s / c_{1} . \\
4 u u^{\prime} & \equiv x x^{\prime}(m+t)^{2}+w w^{\prime}(t-m)^{2} c_{2} / c_{1}+\left(x w^{\prime}+x^{\prime} w\right)\left(t^{2}-m^{2}\right) s / c_{1} . \\
u u^{\prime}+v v^{\prime} & \equiv\left(x x^{\prime}+w w^{\prime} c_{2} / c_{1}\right)\left(m^{2}+t^{2}\right) / 2 \equiv\left(-c_{1} x x^{\prime}-c_{2} w w^{\prime}\right) / c_{3},
\end{aligned}
$$

by (16). Hence

$$
\text { if } A=\left|c_{1} x x^{\prime}+c_{2} w w^{\prime}+c_{3} u u^{\prime}+c_{3} v v^{\prime}\right| \text {, then } A \equiv 0(\bmod p) .
$$

Multiply together the representations of $k p$ given in (3) corresponding to the two solutions in (18):

$$
\begin{aligned}
(k p)^{2}= & A^{2}+c_{1} c_{2}\left(x w^{\prime}-x^{\prime} w\right)^{2}+c_{1} c_{3}\left(x u^{\prime}-x^{\prime} u\right)^{2}+c_{1} c_{3}\left(x v^{\prime}-x^{\prime} v\right)^{2} \\
& +c_{2} c_{3}\left(w u^{\prime}-w^{\prime} u\right)^{2}+c_{2} c_{3}\left(w v^{\prime}-w^{\prime} v\right)^{2}+c_{3}^{2}\left(u v^{\prime}-u^{\prime} v\right)^{2} .
\end{aligned}
$$

This implies that $A \leq k p$.

In order to prove that the two solutions in (18) are essentially the same, one first verifies that (5) through (9) are satisfied. This inchudes actually exhibiting $m$ and $t$. Having thereby justified the expressions for $u$ and $v$ given in (17), one then seeks to show that $A=k p$, so that

$$
x w^{\prime}=x^{\prime} w, \quad x u^{\prime}=x^{\prime} u, \quad x v^{\prime}=x^{\prime} v .
$$


In every case to be considered here, the greatest common divisor $D$ of $c_{2}$ and $c_{3}=c_{4}$ does not divide $k$, and $c_{1}=1$. Then according to (3) $D+x$. Hence $x \neq 0$, so that (21) implies $w / x=w^{\prime} / x^{\prime}, u / x=u^{\prime} / x^{\prime}, v / x=v^{\prime} / x^{\prime}$. Thus if $A=k p$, then $x^{\prime}= \pm x, w^{\prime}= \pm w, u^{\prime}= \pm u$ and $v^{\prime}= \pm v$. That $c_{1} x$ is not divisible by $D$ implies, furthermore, that in (19), $A \neq 0$. Consequently, if $k=1$, it suffices to verify that (5) through (9) hold.

Although the notation here is modeled after that of Dickson, there are differences. If $p \equiv 1(\bmod 5)$, and $(1)$ and $(2)$ are satisfied, choose $r$ such that ord $_{p} r=5$. Set

$$
\begin{array}{ll}
m \equiv\left(2 r-r^{2}+r^{3}-2 r^{4}\right) / 25, & t \equiv\left(r+2 r^{2}-2 r^{3}-r^{4}\right) / 25, \\
s \equiv 5\left(r-r^{2}-r^{3}+r^{4}\right), & s^{2} \equiv 125 .
\end{array}
$$

There is also the form having $k=1, c_{1}=1, c_{2}=c_{3}=c_{4}=5, d_{1}=d_{2}=-d_{3}$ $=-d_{4}=1[2$, Theorem 8]. Set

$$
\begin{array}{ll}
m \equiv\left(r-r^{2}+r^{3}-r^{4}\right) / 5, & t \equiv\left(r+r^{2}-r^{3}-r^{4}\right) / 5, \\
m t \equiv-\left(r-r^{2}-r^{3}+r^{4}\right) / 25 \equiv-s / 25, & s^{2} \equiv 5 .
\end{array}
$$

If $p \equiv 1(\bmod 16)$, then $k=1, c_{1}=1, c_{2}=c_{3}=c_{4}=8, d_{1}=d_{2}=-d_{3}$ $=1, d_{4}=2[3$, p. 236]. (Uniqueness is mentioned there.) 8 is a quadratic residue of $p$. Choose $r$ so that ord $p=16$. Then

$$
\begin{aligned}
s & \equiv 2\left(r^{2}+r^{14}\right), \quad m \equiv\left(r+r^{7}\right) / 4, \quad t \equiv-\left(r^{3}+r^{5}\right) / 4 . \\
s^{2} & \equiv 4\left(r^{4}+2+r^{12}\right) \equiv 8 . \\
m^{2} & \equiv\left(r^{2}+2 r^{8}+r^{14}\right) / 16 \equiv-1 / 8+s / 32 . \\
t^{2} & \equiv\left(r^{6}+2 r^{8}+r^{10}\right) / 16 \equiv\left(-r^{14}-2-r^{2}\right) / 16 \equiv-1 / 8-s / 32 . \\
m t & \equiv-\left(r^{4}+r^{6}+r^{10}+r^{12}\right) / 16 \equiv s / 32 .
\end{aligned}
$$

Hence (9), (14), and (15) are satisfied, and the proof of uniqueness is complete.

If $p \equiv 7(\bmod 16)$, there is a form with $k=1, c_{1}=1, c_{2}=c_{3}=c_{4}=2$, $d_{1}=2, d_{2}=-d_{3}=1, d_{4}=-2[2,(6.1),(6.2)] .2$ is a quadratic residue of $p$. Choose $r \in G F\left(p^{2}\right)$ such that $r^{16}=1$ but $r^{8} \neq 1$. Then $s=r^{2}+r^{14}, m=\left(r+r^{7}\right) / 2$ and $t=\left(r^{3}+r^{5}\right) / 2$ all lie in the ground field. Also

$$
\begin{aligned}
& s^{2}=r^{4}+2+r^{12}=2 \\
& m^{2}=\left(r^{2}-2+r^{14}\right) / 4=-1 / 2+s / 4, \\
& t^{2}=\left(r^{6}-2+r^{10}\right) / 4=-1 / 2-s / 4, \quad m t=-s / 4 .
\end{aligned}
$$

Uniqueness is established.

Now consider the following form for $p \equiv 1(\bmod 60)$ : 


$$
k=c_{1}=1, \quad c_{2}=45, \quad c_{3}=c_{4}=15, \quad d_{1}=d_{2}=-d_{3}=-d_{4}=1
$$

[4, Theorem 2]. Choose $z$ such that $\operatorname{ord}_{p} z=60$. Set $r \equiv z^{12}, R \equiv z^{5}$. Then

$$
\begin{aligned}
s & \equiv 3\left(r-r^{2}-r^{3}+r^{4}\right), \\
m & \equiv\left(R+R^{11}\right)\left(r-r^{2}+r^{3}-r^{4}\right) / 15, \\
t & \equiv\left(R+R^{11}\right)\left(r+r^{2}-r^{3}-r^{4}\right) / 15, \quad s^{2} \equiv 9 \cdot 5 \equiv 45, \\
m^{2} & \equiv\left(R^{2}+2+R^{10}\right)\left(r^{2}+r^{4}+r^{6}+r^{8}-4-2 r^{3}+2 r^{4}+2 r-2 r^{2}\right) / 225 \\
& \equiv 3(-1-4+2 s / 3) / 225 \equiv-1 / 15+2 s / 225 .
\end{aligned}
$$

Similarly,

$$
t^{2} \equiv-1 / 15-2 s / 225, \quad m t \equiv 3\left(-r+r^{2}+r^{3}-r^{4}\right) / 225 \equiv-s / 225,
$$

Uniqueness is proved.

Finally.we present a form for which we have been unable to establish uniqueness. If $p \equiv 1(\bmod 13)$, then $k=16, c_{1}=1, c_{2}=13, c_{3}=c_{4}=26$, $d_{1}=1, d_{2}=-d_{3}=3, d_{4}=-4[4$, Theorem 1]. 13 is a quadratic residue of $p$. Choose $r$ so that ord $p=13$. Define the periods $y_{0} \equiv r+r^{3}+r^{9}, y_{1} \equiv$ $r^{2}+r^{6}+r^{5}, y_{2} \equiv r^{4}+r^{12^{p}}+r^{10}, y_{3} \equiv r^{8}+r^{11}+r^{7}\left[1\right.$, p. 392]. Then $s \equiv y_{0}$ $+y_{2}-y_{1}-y_{3}, m \equiv\left(y_{0}-y_{2}\right) / 13, t \equiv\left(y_{1}-y_{3}\right) / 13$. From the multiplication table for the periods

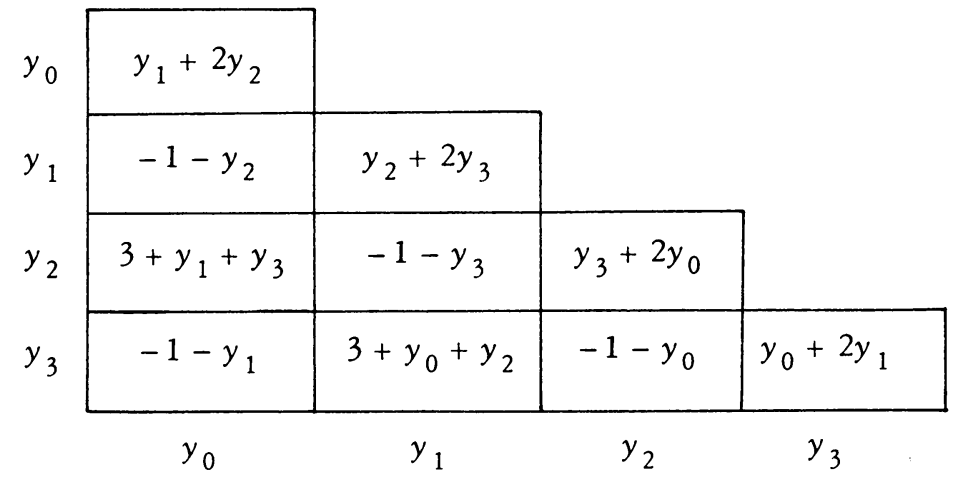

it is easy to verify that

$$
\begin{aligned}
s^{2} & \equiv 20+7\left(y_{0}+y_{1}+y_{2}+y_{3}\right) \equiv 13 \\
m^{2} & \equiv\left(-6+2 y_{0}-y_{1}+2 y_{2}-y_{3}\right) / 13^{2} \\
& \equiv(-61 / 2+3 s / 2) / 13^{2} \equiv-1 / 26+3 s / 338 \\
t^{2} & \equiv-1 / 26-3 s / 338, \\
m t & \equiv\left(y_{0}-y_{2}\right)\left(y_{1}-y_{3}\right) / 13^{2} \equiv\left(-y_{0}+y_{1}-y_{2}+y_{3}\right) / 13^{2} \equiv-s / 13^{2} .
\end{aligned}
$$


Since in this case $k=16$, completing a proof of uniqueness requires showing that $A=16 p$. In other words, by (19), if $A=M p, M$ cannot assume any of the integer values from 1 to 15 . Regarding (3) as a congruence (mod 2) gives $x \equiv w(\bmod 2)$ and $x^{\prime} \equiv w^{\prime}(\bmod 2)$. Hence $x x^{\prime}+13 w w^{\prime}$ is even, so $M$ is even, by (19). According to (20), $M^{2} \equiv 16^{2}(\bmod 13)$. These conditions exclude all possible values of $M$ except 10 . We have been unable to eliminate this possibility.

A computer search of all primes $p \equiv 1(\bmod 13), p<10,000$, revealed no instance of nonunique solutions. We wish to thank the University of Pittsburgh Computer Center for granting access to its IBM 7090/1401 system, partially supported by National Science Foundation grant G-11309.

This research was partially supported by National Science Foundation grants GP-5308 and GP-8973. The second author also received support under a Faculty Research Grant, California State University, Fullerton.

\section{REFERENCE}

1. L. E. Dickson, Cyclotomy, higher congruences, and Waring's problem, Amer. J. Math. 57 (1935), 391-424.

2. R. E. Giudici, J. B. Muskat and S. F. Robinson, On the evaluation of Brewer's character sums, Trans. Amer. Math. Soc. 171 (1972), 317-347. MR 46 \#5249.

3. H. Hasse, Der $2^{n}$-te Potenzcharakter von 2 im Körper der $2^{n}$-ten Einheitswurzeln, Rend. Circ. Mat. Palermo (2) 7 (1958), 185-244. MR 21 \#4143.

4. Y. C. Zee, The Jacobi sums of orders thirteen and sixty and related quadratic decompositions, Math. Z. 115 (1970), 259-272. MR 41 \#6812.

DEPARTMENT OF MATHEMATICS, BAR-ILAN UNIVERSITY, RAMAT-GAN, ISR AEL

DEPARTMENT OF MATHEMATICS, CALIFORNIA STATE UNIVERSITY, FULLERTON, CALIFORNIA 92634 